\title{
Oridonin inhibits metastasis of human ovarian cancer cells by suppressing the mTOR pathway
}

Ye Wang, Zhiling Zhu

Department of Integration of Western and Traditional Medicine, Obstetrics and Gynecology Hospital of Fudan University, Shanghai, China

Submitted: 8 May 2018

Accepted: 16 June 2018

Arch Med Sci 2019; 15 (4): 1017-1027

DOI: https://doi.org/10.5114/aoms.2018.77068

Copyright $\odot 2018$ Termedia \& Banach

\section{Abstract}

Introduction: Oridonin, which is isolated from the Chinese herb Rabdosia rubescens, has been reported to exhibit an anti-tumorous effect on different cancers. In this study, we investigated the molecular mechanism by which oridonin suppresses human ovarian cancer.

Material and methods: The inhibition of oridonin on cell proliferation was assessed by CCK8 assay. Cell cycle and apoptosis were analyzed by flow cytometry, staining with propidium iodide $(\mathrm{PI})$ or annexin-V/PI respectively. The metastasis rate was evaluated using a transwell migration assay. The expression of metastasis-associated genes and mTOR pathway related genes were detected by western blot.

Results: We demonstrated that oridonin suppressed the proliferation and blocked the cell cycle in G1/S phage and induced apoptosis in SKOV3 and A2780 cells $(p<0.01)$. We further found that the mTOR signaling pathway was suppressed by the treatment with oridonin, and the activation of the mTOR pathway attenuated the anti-tumorous effect of oridonin in human ovarian cancer cells, suggesting that the mTOR pathway was involved in the anti-tumorous process of oridonin. Additionally, the activation of the mTOR pathway by an exogenous activator reduced the expression level of FOXP3 $(p<0.01)$, thus providing evidence that FOXP3 is a factor that is necessary for the anti-tumorous effect of oridonin, and is negatively regulated by the MTOR pathway. Conclusions: These results suggested that oridonin suppressed the mTOR signaling pathway, up-regulated the FOXP3 level, and inhibited metastasis of human ovarian cancer cells.

Key words: oridonin, metastasis, mTOR pathway, FOXP3, human ovarian cancer.

\section{Introduction}

As one of the most common gynecological malignant tumors, ovarian cancer carries the highest mortality [1]. Most ovarian cancers were diagnosed at a late stage with metastasis, resulting in a dismal 30\% 5-year survival rate. In the case that ovarian cancer is diagnosed at an early stage, the 5 -year survival rate can be raised to an exciting 90\% [2-4]. Metastasis is a significant factor of the low survival rate of ovarian cancer; therefore, development of more effective drugs to suppress cell growth and metastasis has become the key issue for ovarian cancer therapy.

Traditional herb medicines and herbal derived components are playing increasingly important roles in prevention and treatment of cancers [5-8], such as Astragali radix [9], Codonopsis pilosula [10] and Andro-

\author{
Corresponding author: \\ Zhiling Zhu \\ Department of Integration \\ of Western and \\ Traditional Medicine, \\ Obstetrics and \\ Gynecology \\ Hospital of Fudan \\ University \\ 419 Fangjiao Road \\ 200011 Shanghai, China \\ Phone: 13601882250 \\ E-mail: zhuzhiliifdm@163.com
}


grapholide [11]. Oridonin, an ent-kaurane diterpenoid $\left(\mathrm{C}_{20} \mathrm{H}_{28} \mathrm{O}_{6}\right)$ isolated from the Chinese herb Rabdosia rubescens, has attracted researchers' attention for its various pharmacological activities in recent years, such as anti-tumor, anti-bacterial, and anti-inflammatory properties [12-14]. It has been reported that oridonin inhibited growth and induced apoptosis in various types of tumors [1518]. For human ovarian cancers, previous studies showed that oridonin inhibited the proliferation of two types of cell lines that are sensitive or insensitive to the chemotherapeutic drug paclitaxel [19], and reversed cisplatin drug resistance effectively [20]. In addition, Wang et al. found that oridonin not only induced apoptosis, but also inhibited the metastasis and invasion of human breast cancer cells [21]. The Notch signaling pathway was claimed to play an important role in the inhibition of metastasis induced by oridonin [22, 23]. For pancreatic cancer, oridonin was also reported to inhibit the metastasis and epithelial-mesenchymal transition [24]. However, the mechanism underlying the anti-metastasis effect of oridonin remains largely unknown.

Oridonin has been reported to suppress cell proliferation in ovarian cancer and inhibit metastasis and invasion in human breast cancer cells. We hypothesized that oridonin has an antitumoral effect on human ovarian cancer cells in several processes, including cell proliferation, apoptosis and metastasis. The aims of the current study were to (i) investigate the effect of oridonin on proliferation, apoptosis, and metastasis in human ovarian cancer cells, and (ii) explore the molecular mechanism of the antitumoral effect of oridonin on human ovarian cancer cells.

\section{Material and methods}

\section{Cell culture and transfection}

SKOV3 cells were grown in McCoy's 5A (Modified) Medium (Gibco), and A2780 cells were grown in RPMI-1640 Medium (Hyclone), under $5 \% \mathrm{CO}_{2}$ at $37^{\circ} \mathrm{C}$. The two media above were supplemented with $10 \%$ fetal bovine serum (Hyclone), $100 \mathrm{U} / \mathrm{ml}$ penicillin and $100 \mu \mathrm{g} / \mathrm{ml}$ streptomycin (Gibco). Cells were plated at $2 \times 10^{5}$ cells per well in 6 -well plates for siRNA transfection. Transfection was performed using Lipofectamine 3000 (Invitrogen), following the manufacturer's instructions. Cells were transfected with siRNAs at a final concentration of $100 \mathrm{nM}$. siRNAs were ordered from Genepharma (Shanghai, China). FOXP3 siRNA: 5'-GGCGGACCAUCUUCUGGAUdTdT-3'.

\section{Preparation of oridonin solution}

Oridonin was bought from Abcam. Oridonin powder was dissolved in DMSO (Sigma) at $50 \mathrm{mM}$ and stored at $-80^{\circ} \mathrm{C}$. Storage oridonin solution was diluted to $10 \mathrm{mM}$ before use.

\section{Western blot}

Cells were harvested, washed with phosphate buffered saline (PBS) and lysed with lysis buffer (Sigma). The protein concentration of cell lysate was determined using the Bicinchoninic acid (BCA) protein assay (Invitrogen). Forty micrograms of proteins were resolved by electrophoresis on $8 \%$ or $10 \%$ Tris-glycine polyacrylamide gels and transferred to polyvinylidene fluoride (PVDF) membranes. The membranes were blocked in 2.5\% skimmed milk for 1 hour and incubated overnight with the primary antibody to MMP-2, FAK, p-mTOR (Ser2448), mTOR (Cell Signaling, $1: 1000$ dilution), MMP-9, FOXP3 (abcam, $1: 1000$ dilution) or GAPDH (Bioworld, 1 : 2000 dilution) at $4^{\circ} \mathrm{C}$. After washing three times, the membranes were incubated with the second antibody (ZSGBBio, $1: 4000$ dilution) for $2 \mathrm{~h}$ at room temperature. Blots of proteins were detected using a chemiluminescence detection system (CWBIO).

\section{Cell proliferation and cytotoxicity assay}

Cells were plated at $3 \times 10^{3}$ cells per well in a 96-well plate $24 \mathrm{~h}$ before treatment. After treatment, cell viability was assessed using a CCK-8 Kit (Dojindo) following the manufacturer's instructions. In brief, CCK-8 reagent was diluted in serum free medium in advance (1: 10). Medium of samples was removed from the 96-well plate. Cells were washed with PBS, then CCK 8 reagent $(100 \mu \mathrm{l} /$ well) was added into the 96 -well plate. Samples were incubated at $37^{\circ} \mathrm{C}$, and the intensity of absorbance was assessed using a Multimode Reader after $2 \mathrm{~h}$.

\section{Transwell assay}

Cells were suspended in serum free medium containing $0.1 \% \mathrm{BSA}$, and placed in transwell inserts (Corning Life Sciences) at a concentration of $1 \times 10^{5}$ cells per insert. The final volume of medium is $200 \mu \mathrm{l}$ for each transwell insert. Put transwell inserts in a 24-well plate containing complete medium. After $12 \mathrm{~h}$ ' incubation, erase cells in the inner side of the membrane. Migrated cells were fixed with $4 \%$ paraformaldehyde and stained with crystal violet $(0.1 \%)$. Then remove the membrane and count cells. Images were taken of five areas of the membrane. An average cell count of the five images was then used in statistical analysis.

\section{Cell apoptosis assay}

Apoptotic cells were assessed using an ApoDETECT annexin V-FITC apoptosis detection kit 
(Sigma-Aldrich) by flow cytometry following the kit manual. Cells treated with different concentrations of oridonin were digested, washed twice with cold PBS, and resuspended in binding buffer. Add $5 \mu \mathrm{l}$ of FITC-labeled annexin $\mathrm{V}$ in $190 \mu \mathrm{l}$ of cell suspension, mix gently, then add $5 \mu$ l propidium iodide (PI) solution. Incubate cells in dark for $10 \mathrm{~min}$ at room temperature. Cells were analyzed on a flow cytometer (BD Biosciences).

\section{Cell cycle assay}

SKOV 3 and A2780 cells were plated at the density of $5 \times 10^{5}$ cells in $6 \mathrm{~cm}$ dishes and treated with different concentrations of oridonin. After $24 \mathrm{~h}$, cells were collected and fixed in ice-cold $70 \%$ ethanol (v/v) overnight at $4^{\circ} \mathrm{C}$. Wash cells with PBS and collect cells, resuspend cells in PBS containing $50 \mu \mathrm{g} / \mathrm{ml} \mathrm{PI}, 100 \mu \mathrm{g} / \mathrm{ml}$ RNase A, 0.2\% Triton $\mathrm{X}-100$ and stain for $30 \mathrm{~min}$ in the dark, at $4^{\circ} \mathrm{C}$. Then cells were analyzed by a flow cytometer (BD Biosciences). The results were calculated by the Flowjo software.

\section{Statistical analysis}

All data are shown as mean \pm SEM. Statistical analysis was performed with Student's $t$-test to evaluate single-factor differences between two sets of data, or with ANOVA followed by the Bonferroni post-hoc test for multiple comparisons.

\section{Results}

\section{Oridonin inhibited cell proliferation and induced cell cycle arrest in human ovarian cancer cells}

SKOV3 and A2780 cells were treated independently with oridonin at different concentrations for 24-72 h. After treatment, we used CCK8 assay to assess the cell viability of different cell lines. The proliferation of SKOV3 cells was almost abolished by $15 \mu \mathrm{M}$ oridonin (Figure $1 \mathrm{~A}$ ). The suppression of cell viability of A2780 was also strengthened along with the concentration of oridonin treatment (Figure $1 \mathrm{~B}$ ). For the human normal liver cells, HL-7702 cells, only weak suppression of proliferation was detected compared with SKOV3 and A2780 cells (Figure $1 \mathrm{C}$ ). These results showed that cancer cells could be more sensitive to the treatment of oridonin compared with normal cells, indicating that oridonin is a potential anti-cancer drug, which is hypotoxic to normal tissue.

To further study whether the proliferation inhibition is related to cell cycle arrest, we assessed the cell progression of human ovarian cancer cells treated with different concentrations of oridonin by flow cytometry. In SKOV3 cells, treatment with oridonin $(5-20 \mu \mathrm{M})$ induced an increased S phage cell population, from $35.85 \%$ to $43.17 \%(20 \mu \mathrm{M})$, while the cell population of $\mathrm{G} 1$ phage had no obvious increase (Figure 1 D, Table I). In A2780 cells, we observed that the $\mathrm{G} 1$ phage cell population increased from $70.75 \%$ to $87.10 \%(20 \mu \mathrm{M})$, while the cell population of $S$ phage and $G 2$ phage decreased (Figure $1 \mathrm{E}$, Table I). Combining the results above, the cell progression of human ovarian cancer cells was affected by oridonin, and the cell cycle was arrested in G1/S phage.

\section{Oridonin induced human ovarian cancer cells apoptosis}

To determine whether oridonin induces cell apoptosis in human ovarian cancer cells other than the inhibition of cell proliferation, we examined the apoptosis of human ovarian cancer cell lines after $12 \mathrm{~h}$ of oridonin treatment $(5-20 \mu \mathrm{M})$ by flow cytometry. As Figure 2 shows, in SKOV3 (Figures 2 A, C) and A2780 cells (Figures 2 B, D), both early and late apoptosis rates were increased by oridonin treatment in two cell lines, in a dose-dependent manner (Figures 2 A, B). When treating human ovarian cancer cells with $10 \mu \mathrm{M}$ or $20 \mu \mathrm{M}$ oridonin, we observed significant apoptosis in SKOV3 and A2780 cells (Figures 2 C, D). Thus, oridonin induced cell apoptosis in human ovarian cancer cells.

\section{Oridonin inhibited metastasis of human ovarian cancer cells through the mTOR pathway}

Metastasis plays an important role in the development of cancer, and it is also a key standard in the assessment of new anti-cancer drugs. To evaluate the effect of oridonin on the metastasis of human ovarian cancer cell lines, we performed a transwell assay to assess the metastasis rates of oridonin-treated SKOV 3 cells $(1.25-5 \mu \mathrm{M})$. Twelve hours after oridonin treatment, erasing the cells in the inner side of the membrane, migrated cells were fixed and stained (Figure $3 \mathrm{~A}$ ). Cell counting results showed that the treatment with oridonin suppressed the metastasis of SKOV3 cells significantly in the concentration of $2.5 \mu \mathrm{M}$ and $5 \mu \mathrm{M}$ (Figure 3 B). We also assessed the expression level of matrix metalloproteinase-2 (MMP-2), matrix metalloproteinase-9 (MMP-9) and focal adhesion kinase (FAK) by western blot. The expression of these metastasis-related factors decreased after treatment with oridonin in both SKOV3 and A2780 cells (Figures 3 C, D). These data indicated that oridonin not only inhibited the survival and proliferation, but also suppressed the metastasis of human ovarian cancer cells.

Additionally, we found that the mTOR pathway was suppressed by oridonin treatment. With 

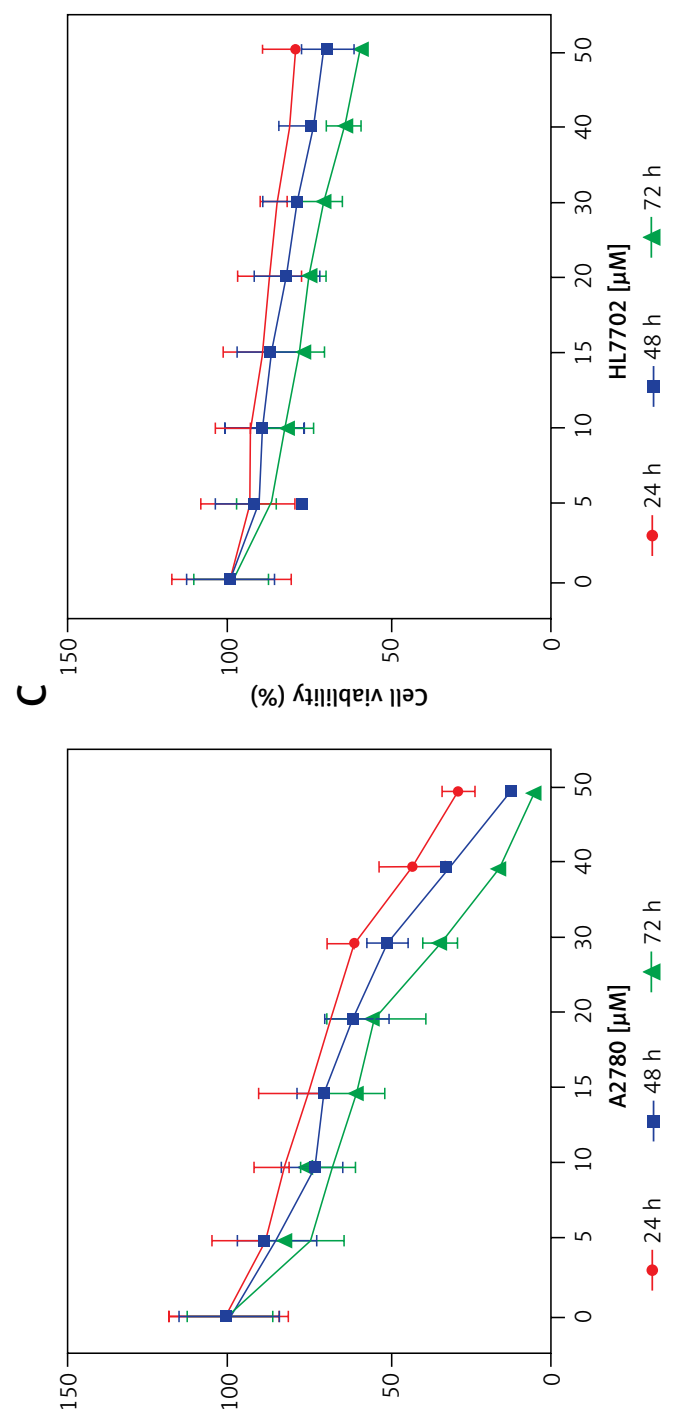

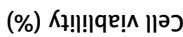

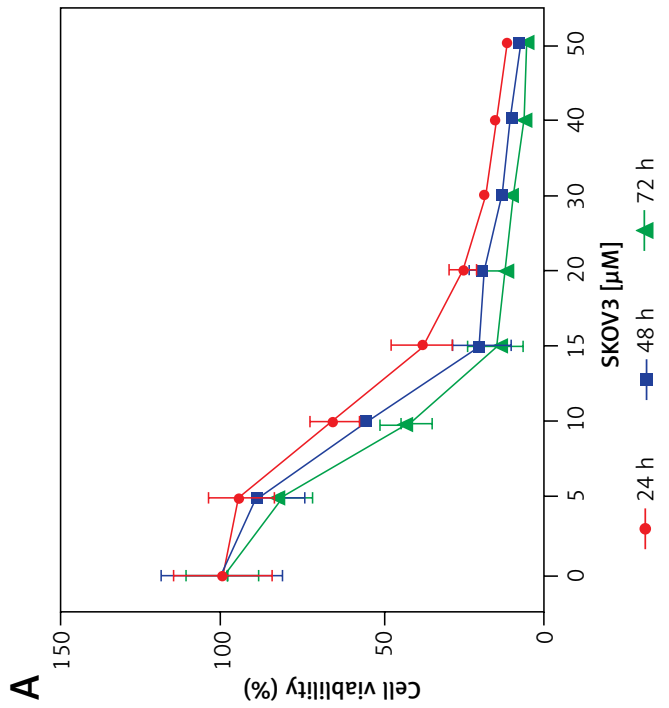

(\%) К‼!!qए!^ ॥əכ
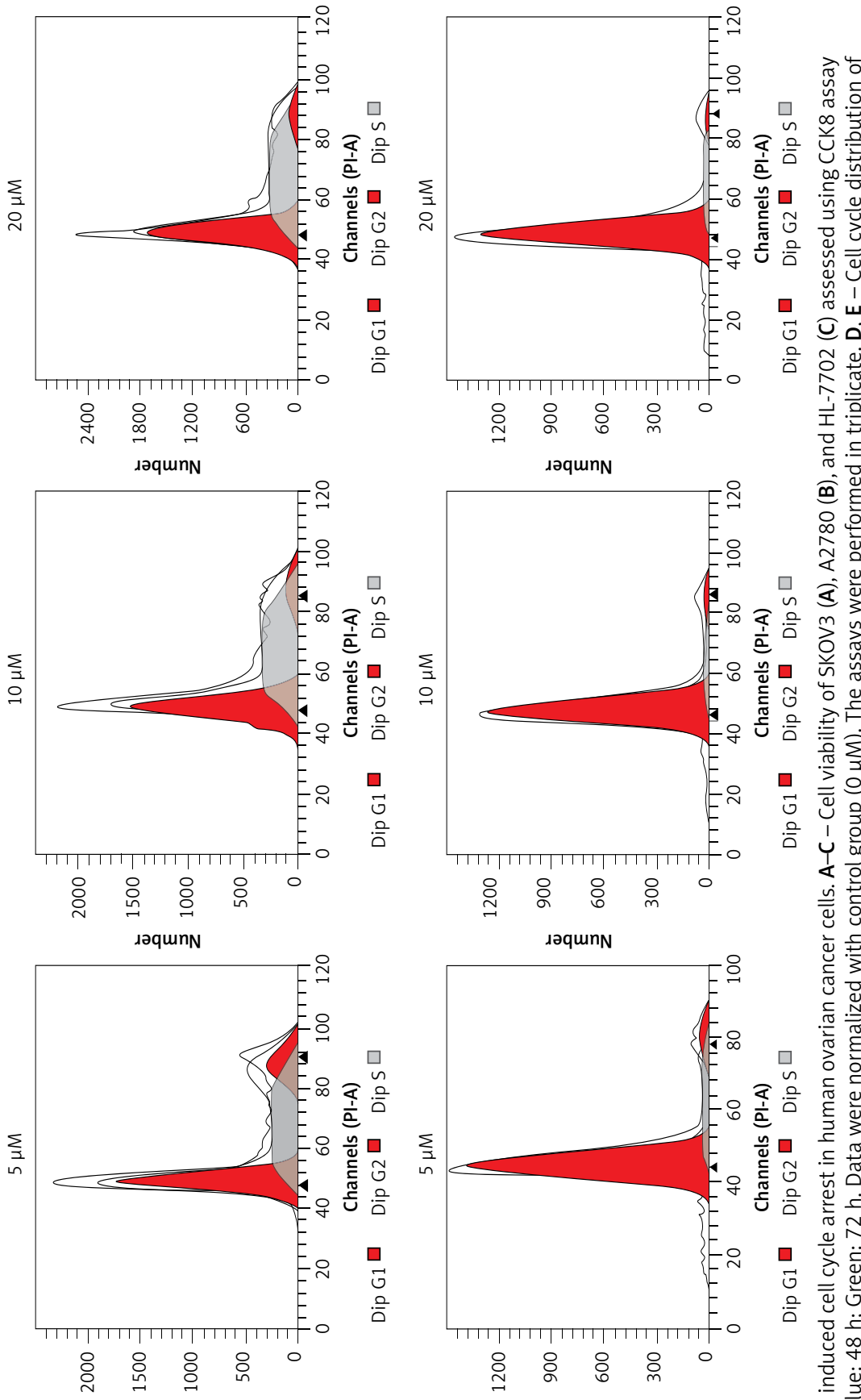

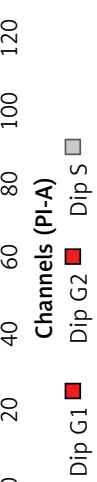

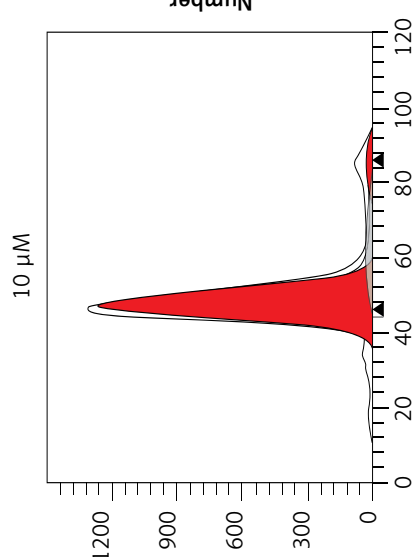

बิ

○ ᄒ̀

ํํํ

的安离 交言管 崩 $8 \frac{n}{0}$ 는 टٓ पे 它 은.

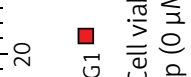

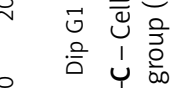
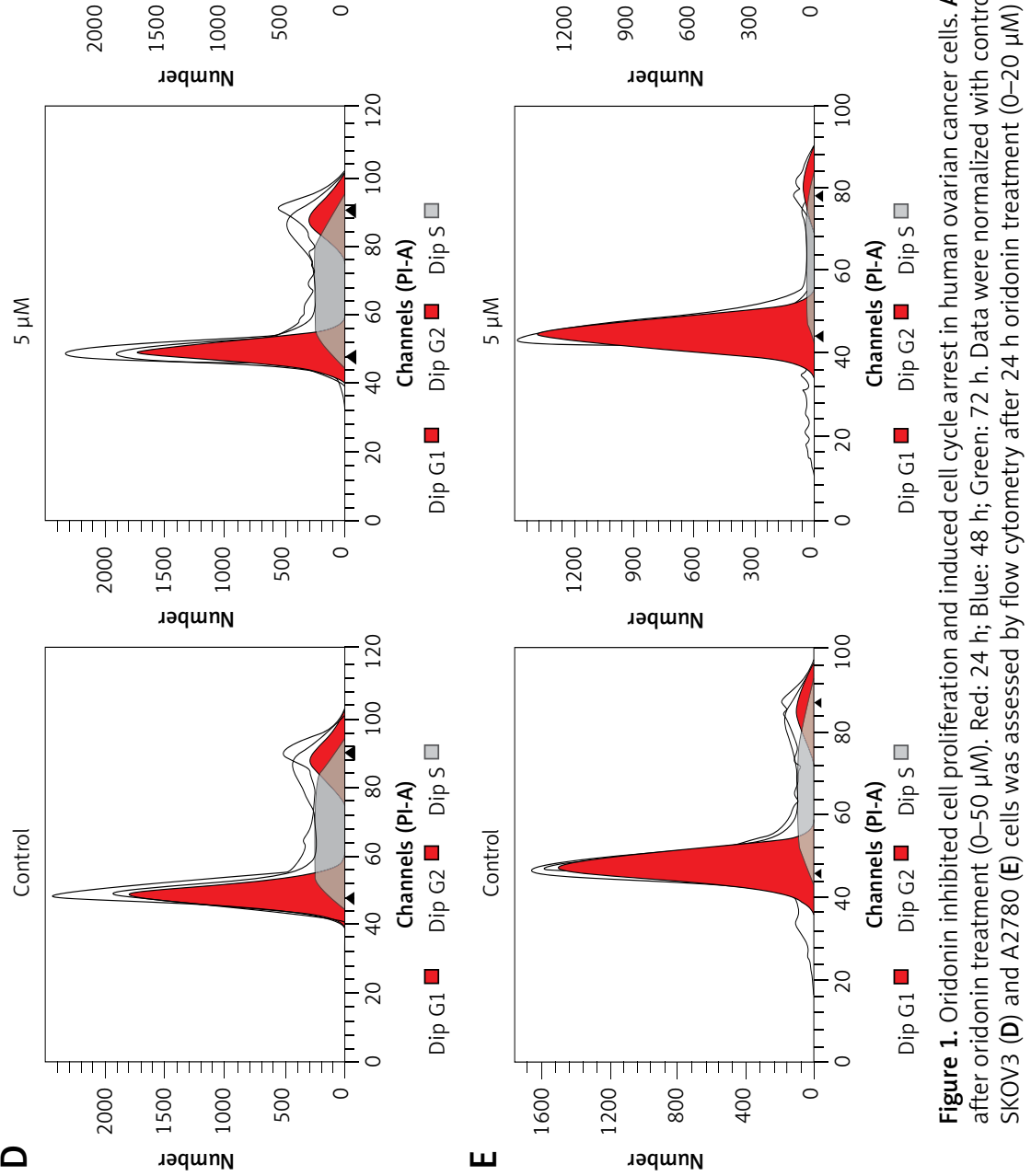
A

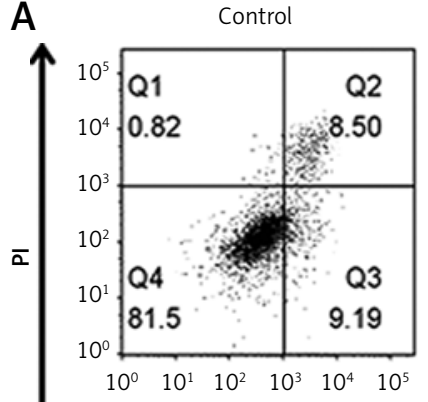

$5 \mu \mathrm{M}$

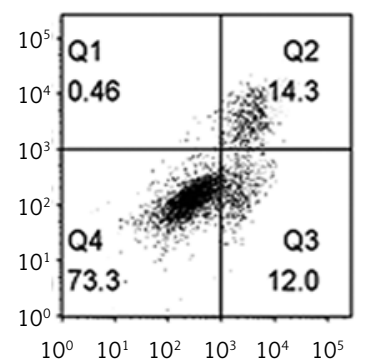

$10 \mu \mathrm{M}$

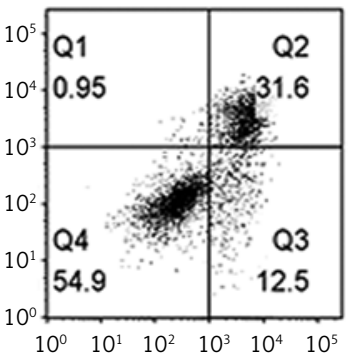

$20 \mu M$

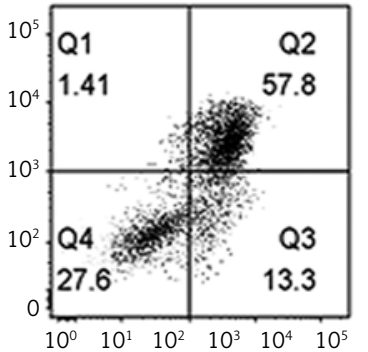

Annexin- $V$

B

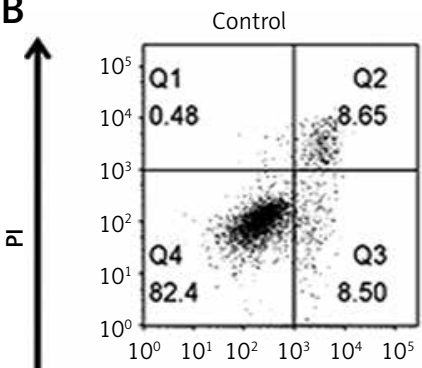

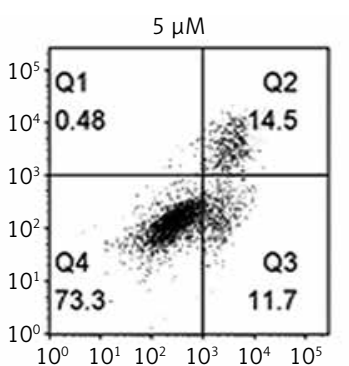

$20 \mu \mathrm{M}$

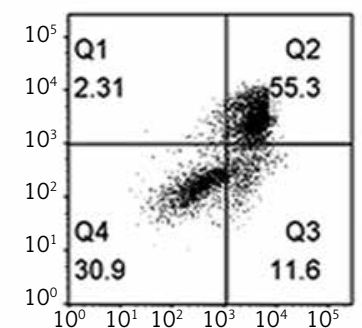

Annexin-V

C

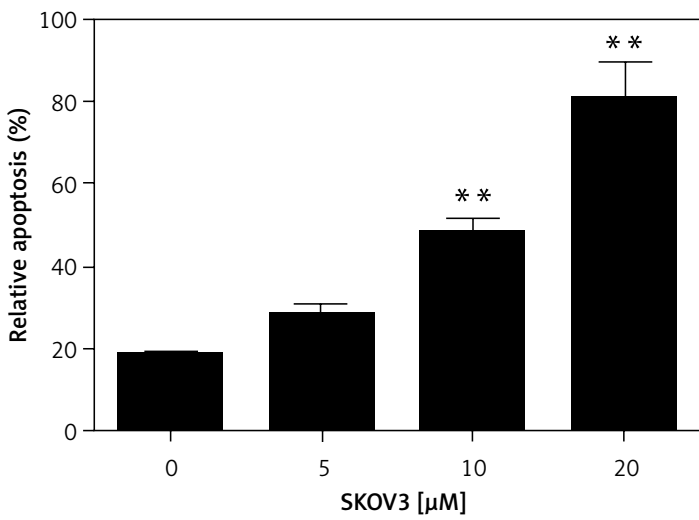

D

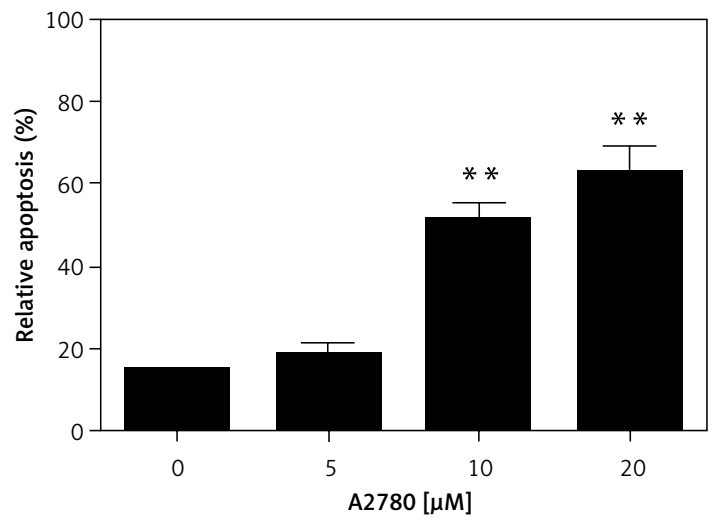

Figure 2. Oridonin induced apoptotic cell death in human ovarian cancer cells. A, B - Representative images of flow cytometry analysis in SKOV3 (A) and A2780 (B) cells. Human ovarian cancer cells were treated with oridonin $(0-20 \mu \mathrm{M})$ for $12 \mathrm{~h}$, then stained with annexin V-FITC/PI, and analyzed by flow cytometry. The early and late apoptosis was quantified and indicated in Q3 and Q2 gates, respectively. C, D - Plot of the percentage of apoptotic cells of SKOV3 (C) and A2780 (D)

Results are presented as the mean \pm SEM of three independent experiments. ${ }^{* \star} P<0.01$

a consistent level of total mTOR expression, the level of $\mathrm{p}$-mTOR decreased along with the concentration of oridonin treatment (Figures 3 C, D). In order to illuminate whether this pathway participated in the anti-metastatic process induced by oridonin, we treated human ovarian cancer cells with an activator of the mTOR pathway, 3BDO, and found that the level of p-mTOR was increased in both SKOV3 (Figure 4 A) and A2780 (Figure 4 B) cells. Using a transwell assay, we assessed the metastasis rate of SKOV3 cells with treatment with oridonin and/or 3BDO. The metastasis rate rose after treatment with only 3BDO for $12 \mathrm{~h}$ (Figure $4 \mathrm{C})$. For the group co-treated with oridonin and $3 \mathrm{BDO}$, the metastasis rate was reduced compared with the $3 \mathrm{BDO}$ only group. Also the pretreatment with 3BDO suppressed the anti-metastatic effect of oridonin in SKOV3 cells (Figure 4 C). Therefore, oridonin inhibited metastasis of human ovarian cancer cells via suppressing the mTOR pathway.

On the other hand, it is unexpected that we also found that FOXP3 was up-regulated when SKOV3 
A

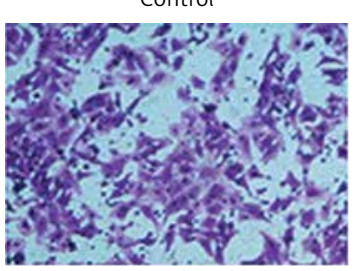

$2.5 \mu \mathrm{M}$

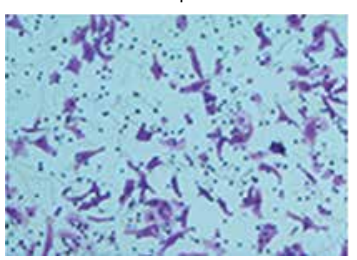

C
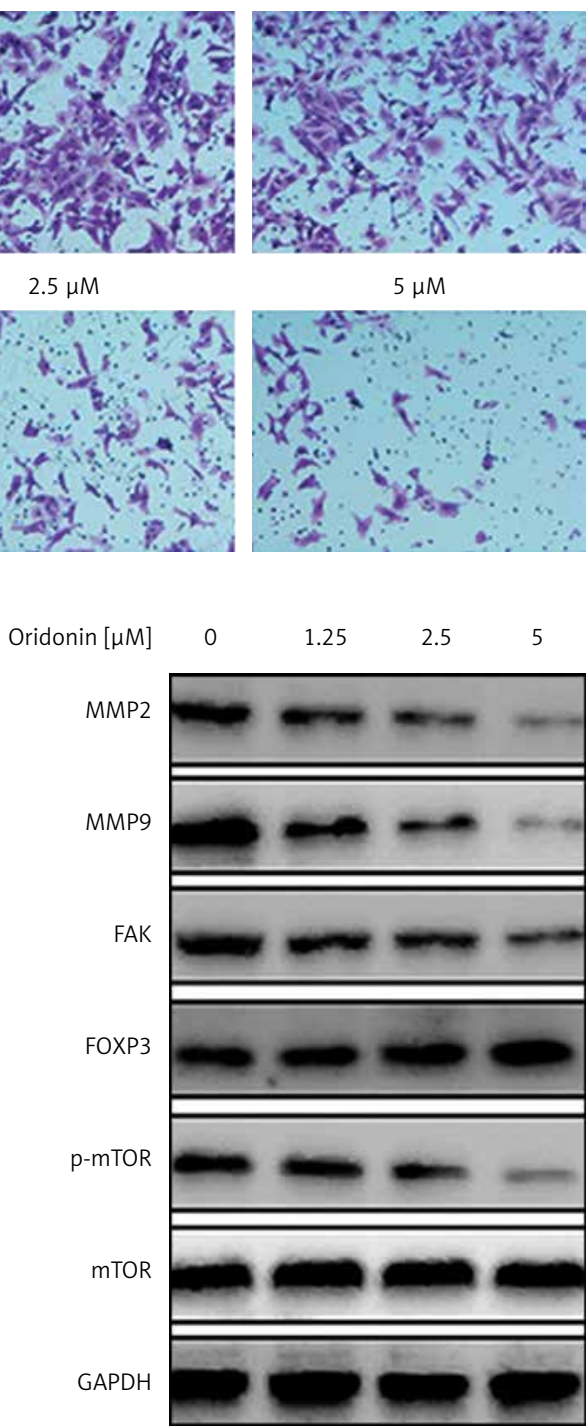

$5 \mu \mathrm{M}$

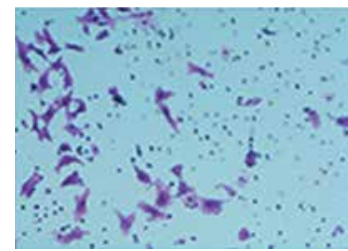

B

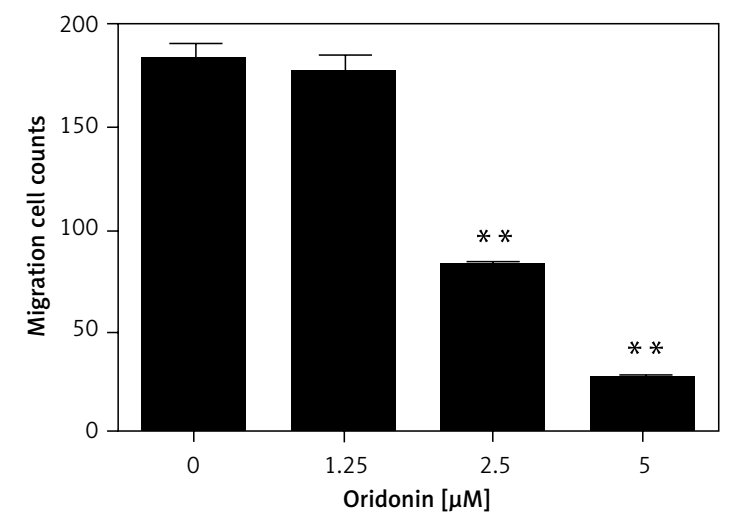

D

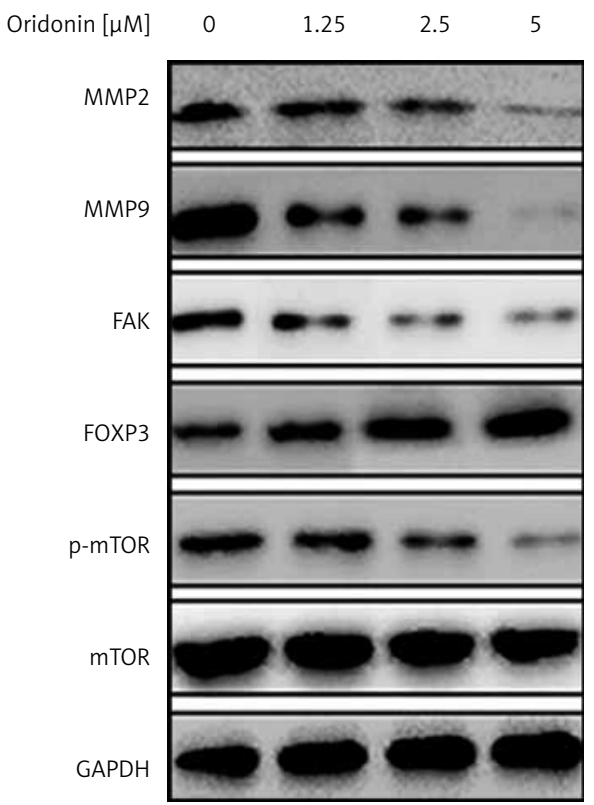

Figure 3. Oridonin suppressed the metastasis of human ovarian cancer cells. A - Representative images of migrated SKOV 3 cells in transwell assay. SKOV 3 cells were treated with oridonin $(0-5 \mu \mathrm{M})$. After $12 \mathrm{~h}$, the cells in the inner side of the membrane were erased, and migrated cells were fixed and stained. B - Plot of the metastasis rate of SKOV3 cells in transwell assay. ${ }^{* *} P<0.01$. C, D - Western blot analysis of MMP-2, MMP-9, FAK, FOXP3, p-mTOR and mTOR genes in SKOV3 (C) and A2780 (D) cells treated with oridonin (0-5 $\mu \mathrm{M})$. GAPDH was used as an internal control

and A2780 cells were treated with oridonin, and the FOXP3 expression level increased along with the raised concentration of oridonin (Figures $3 \mathrm{C}$, D). FOXP3 is a member of the forkhead/winged-helix family of transcriptional regulators, and has been demonstrated to play important roles in the development and function of regulatory $T$ cells [25-28]. In addition, studies have recently shown that FOXP3 is also expressed in some tumor cells, and influenced the development of different cancers [29-34]. To clarify the correlation between the up-regulation of FOXP3 and the suppression of metastasis, we performed experiments to investigate whether the low level of FOXP3 influences the metastasis of human ovarian cancer cells. We designed
siRNA targeting FOXP3, transfected siRNA for $24 \mathrm{~h}$ in SKOV3 and A2780 cells, added $2.5 \mu \mathrm{M}$ oridonin, treated for $12 \mathrm{~h}$, and assessed the expression levels of MMP-2, MMP-9, FOXP3, mTOR and p-mTOR by western blot. We found under the condition of a lower level of FOXP3 that the anti-metastatic effect of oridonin was suppressed. The suppression of MMP-2 and MMP-9 expression was rescued partially in both SKOV3 and A2780 cells (Figures 5 A, B). At the same time, we performed a transwell experiment to assess the metastasis rate of SKOV3 cells co-treated with SIRNA targeting FOXP3 and oridonin. Cells were plated $24 \mathrm{~h}$ after the siRNA transfection. Twelve hours after oridonin treatment, we found that cells transfected with FOXP3 siRNA had 
a higher metastasis rate compared with the cells transfected with NC siRNA (Figures 5 C, D). These results indicated that FOXP3 plays an important role in the anti-metastatic process of oridonin.

Meanwhile, we found that siRNA targeting FOXP3 did not disrupt the level of p-mTOR or mTOR expression (Figures $5 \mathrm{~A}, \mathrm{~B}$ ), while, with the treatment with 3BDO, we observed that FOXP3 was down-regulated along with the activation of the mTOR pathway (Figures 4 A, B). Thus, FOXP3 was negatively regulated by the mTOR pathway. Combine with the former results, oridonin inhibited the mTOR pathway, and then up-regulated the downstream gene FOXP3, and induced suppression of metastasis.

\section{Discussion}

In this study, we confirmed the hypothesis that oridonin has an antitumoral effect on human ovarian cancer cells in several processes, includ-
Table I. Oridonin induced cell cycle arrest in human ovarian cancer cells

\begin{tabular}{|lcccc|}
\hline Variable & Groups & G1 (\%) & S (\%) & G2 (\%) \\
\hline SKOV3 & Control & 49.43 & 35.85 & 14.72 \\
\cline { 2 - 5 } & Oridonin $5 \mu \mathrm{M}$ & 47.70 & 36.51 & 15.78 \\
\cline { 2 - 5 } & Oridonin $10 \mu \mathrm{M}$ & 49.13 & 44.08 & 6.97 \\
\cline { 2 - 5 } & Oridonin $20 \mu \mathrm{M}$ & 50.58 & 43.17 & 5.43 \\
\hline \multirow{2}{*}{ 2780 } & Control & 70.75 & 20.40 & 8.84 \\
\cline { 2 - 5 } & Oridonin 5 $\mu \mathrm{M}$ & 84.62 & 8.93 & 6.42 \\
\cline { 2 - 5 } & Oridonin $10 \mu \mathrm{M}$ & 85.16 & 10.28 & 4.56 \\
\cline { 2 - 5 } & Oridonin $20 \mu \mathrm{M}$ & 87.10 & 9.48 & 3.43 \\
\hline
\end{tabular}

ing cell proliferation, apoptosis and metastasis. We also found that oridonin inhibited the mTOR pathway, and upregulated FOXP3 expression level.
A

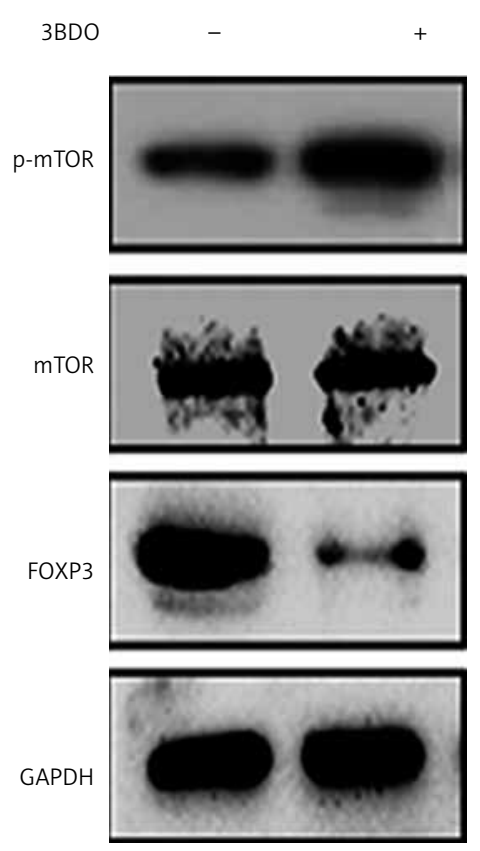

C

Oridonin 3BDO

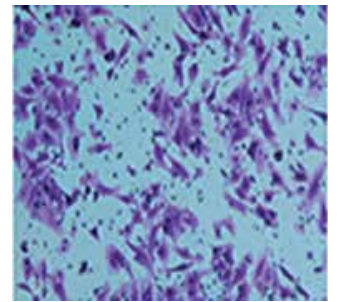

B
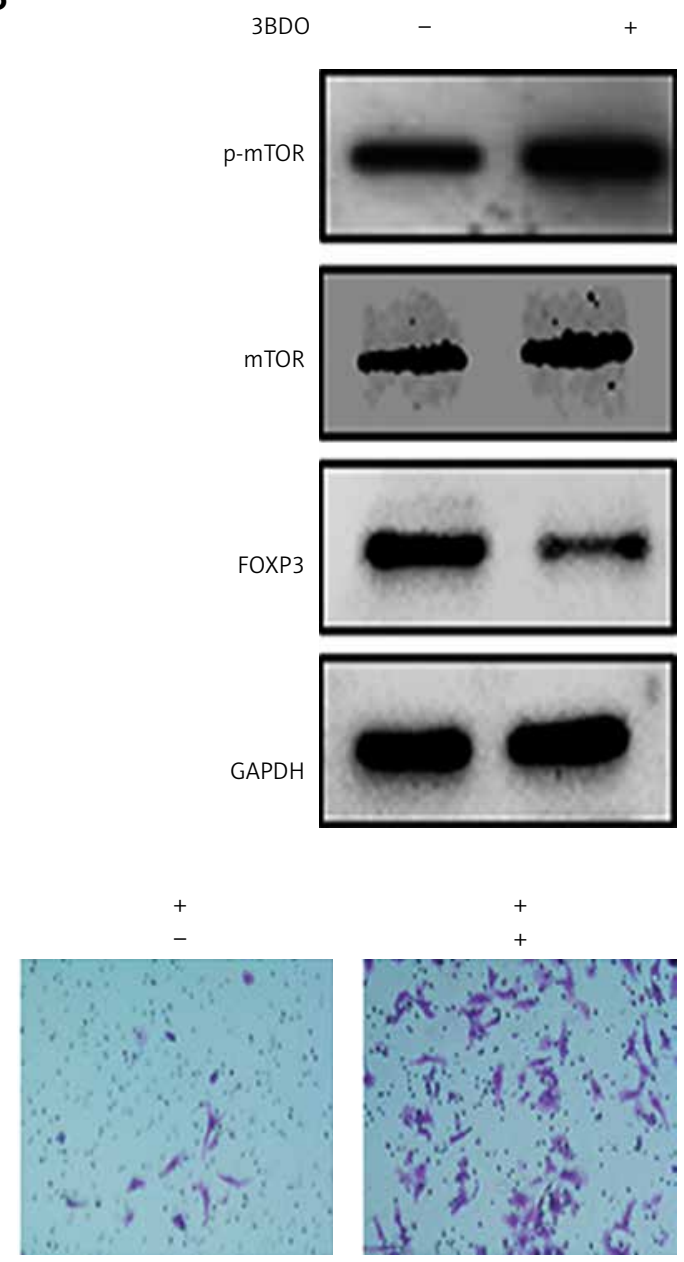

Figure 4. Activation of mTOR pathway attenuated the anti-metastatic effect of oridonin. A, B - Western blot analysis of p-mTOR, mTOR and FOXP3 genes in SKOV3 (A) and A2780 (B) cells treated with 3BDO (30 $\mu$ M). GAPDH was used as an internal control. C - Representative images of migrated SKOV 3 cells in transwell assay. SKOV 3 cells were pretreated with or without $3 \mathrm{BDO}(30 \mu \mathrm{M})$ for $12 \mathrm{~h}$, then oridonin was added $(0$ or $5 \mu \mathrm{M})$. After $12 \mathrm{~h}$, the cells in the inner side of the membrane were erased, and migrated cells were fixed and stained 
A

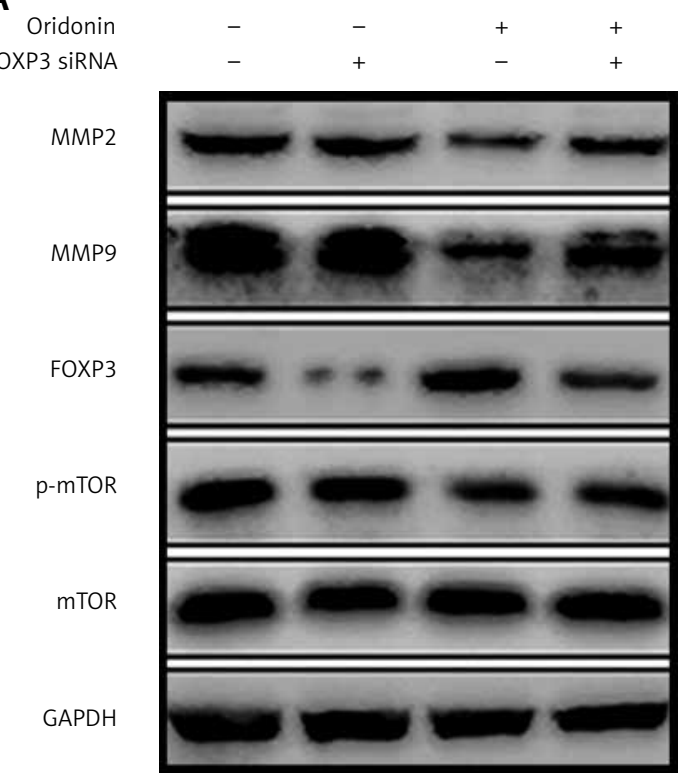

C

Oridonin FOXP3 SIRNA
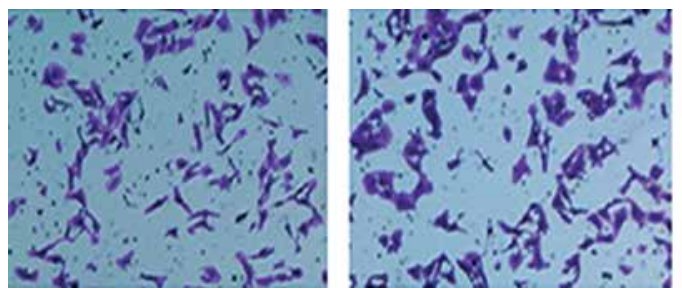

D

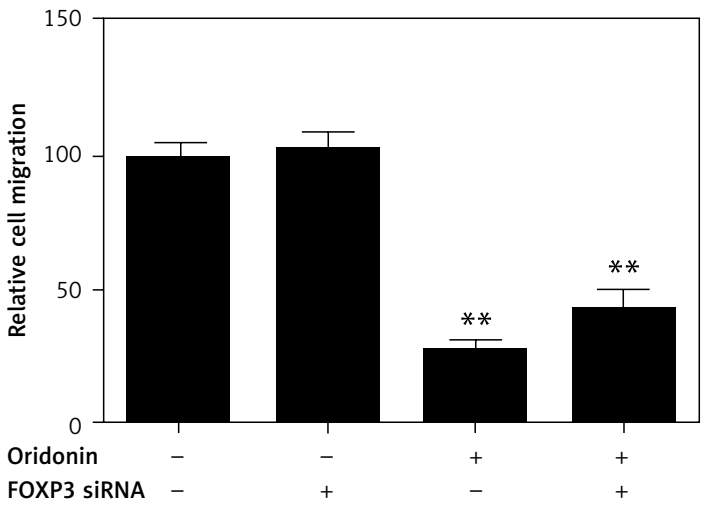

We found that oridonin exerted strong inhibition of the proliferation of human ovarian cancer cells SKOV 3 and A2780, but only showed weak suppression of the proliferation of human liver cells $\mathrm{HL}$ 7702. It could be a sign that human ovarian cancer cells are more sensitive to oridonin, compared with normal cells. This phenomenon indicated that oridonin can be a new effective, specific and hypotoxic anti-cancer drug, which is less lethal for normal tissue during the treatment of cancer.

We also observed that oridonin blocked the cell cycle of human ovarian cancer cells in G1/S
B
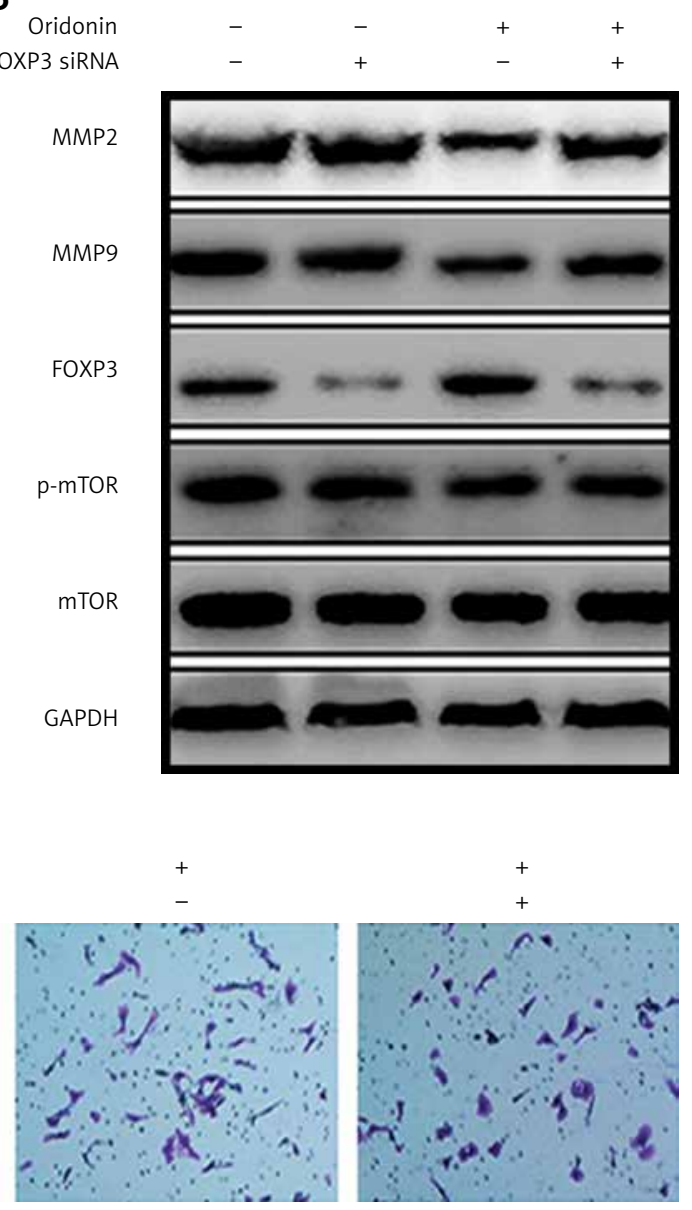

Figure 5. Low level of FOXP3 weakened the antimetastasis effect of oridonin. A, B - Western blot analysis of MMP-2, MMP-9, FOXP3, p-mTOR and mTOR genes in SKOV3 (A) and A2780 (B) cells co-treated with oridonin and FOXP SiRNA. SKOV3 cells were transfected with or without FOXP siRNA $(50 \mathrm{nM})$ for $24 \mathrm{~h}$, then oridonin was added ( 0 or $2.5 \mu \mathrm{M})$. After $12 \mathrm{~h}$, cells were harvested for western blot. GAPDH was used as an internal control. C - Representative images of migrated SKOV3 cells co-treated with oridonin and FOXP siRNA in transwell assay. D - Plot of the metastasis rate of SKOV 3 cells in transwell assay. ${ }^{* *} P<0.01$

phage. Furthermore, oridonin induced significant apoptosis of human ovarian cancer cells. These results supported our former result that oridonin inhibits the proliferation of human ovarian cancer cells (Figure 1).

Other than the effect on cell proliferation and apoptosis, oridonin reduced the metastasis rate of human ovarian cancer cells observably. We detected obvious reduction of the expression of MMP-2 and MMP-9, which are closely related to cancer metastasis, in the cells treated with oridonin. These results showed the anti-tumorous effect of 
oridonin in several aspects: inhibition of proliferation, induction of apoptosis and suppression of metastasis of cancer cells.

The main causes of the high mortality of ovarian cancer are the early metastasis, early infiltration and the resistance to chemotherapy drugs. As the biggest obstacle to curing cancers, metastasis has been one research hotspot for a long time. Several Chinese medicines were reported to have a therapeutic effect on ovarian cancer, such as Cryptotanshinone [35], Scutellaria barbata D. Don [36], Hedyotis diffusa Willd [37], Osthole [38], and others. Previous research also showed that oridonin has anti-tumorous properties in several kinds of tumors [10-13], but the molecular mechanism remains unclear.

In this study, we focused on the anti-cancer effect of oridonin, and found that the mTOR pathway was partially blocked by oridonin treatment. We then performed experiments to activate the mTOR pathway in human ovarian cancer cells using 3BDO. Activation of the mTOR pathway weakened the anti-metastasis function of oridonin treatment. We also found that FOXP3 participated in this process. The function of FOXP3 has been widely studied in T-cell related immune therapy of tumors $[29,39]$. FOXP3 expression in tumors can also regulate the growth, apoptosis [40] or metastasis of tumor cells. Yang et al. claimed that FOXP3 promotes tumor growth and metastasis by activating the Wnt/ $\beta$-catenin signaling pathway and EMT in non-small cell lung cancer [41], while FOXP3 suppresses breast cancer metastasis through the down-regulation of CD44 [42]. Previous research found that in T-cells, the PI3K-Akt-mTOR signaling pathway prevents induction of FOXP3 expression via Foxo factors [43-46], which is consistent with our conclusion in human ovarian cancer cells. In this study, the expression of FOXP3 was induced by oridonin treatment, and siRNA targeting FOXP3 influenced the anti-metastasis effect of oridonin. Under oridonin treatment, FOXP3 was up-regulated while the MTOR pathway was suppressed. It suggests that FOXP3 could be negatively regulated by the mTOR pathway. As evidence supporting this speculation, FOXP3 was down-regulated when the mTOR pathway was activated by $3 \mathrm{BDO}$ (Figures $4 \mathrm{~A}, \mathrm{~B}$ ). These results suggested that the mTOR pathway and FOXP3 played important roles in the anti-metastatic regulatory process of oridonin. Oridonin inhibited the mTOR pathway, and then increased the FOXP3 level, down-regulated metastasis-related factors such as MMP-2 and MMP-9, and suppressed the metastasis of human ovarian cancer cells.

When the SKOV3 cells were treated with FOXP3 siRNA only, we did not detect a significant rise of MMP-2 or MMP-9 expression level (Figures $5 \mathrm{~A}, \mathrm{~B}$ ). The reduction of FOXP3 up-regulated MMP-2 and
MMP-9 expression levels during siRNA/oridonin co-treatment, but not in the condition without oridonin. The reason for this phenomenon may be that FOXP3 is a necessary factor in the anti-metastatic process of oridonin, but a low level of FOXP3 is not sufficient to repress the metastasis independently when cells were not exposed to oridonin. The molecular mechanism of oridonin suppressing the metastasis of human ovarian cancer still remains to be further clarified.

The present study is limited by the lack of evidence in other human ovarian cancer cell lines and in animal models. Experiments were conducted in two human ovarian cancer cell lines, SKOV3 and A2780. Further confirmation in other ovarian cancer cell lines and in a mouse tumor model are needed to investigate the potential applicability of oridonin in the treatment of different types of ovarian cancer. Additionally, our results showed that the mTOR pathway and FOXP3 are involved in the antitumoral process, but how oridonin suppressed the mTOR pathway, and then up-regulated FOXP3, remains unclear. Thus, we need further exploration of the molecular mechanism of the antitumoral effect of oridonin on ovarian cancer in future research.

In conclusion, we found that oridonin suppressed the proliferation, blocked the cell cycle in G1/S phage, and induced cell apoptosis in human ovarian cancer cells. Moreover, through suppressing the mTOR signaling pathway, oridonin inhibited the metastasis of human ovarian cancer cells, a process in which FOXP3 played an important role.

\section{Conflict of interest}

The authors declare no conflict of interest.

\section{References}

1. Gut P, Waligórska-Stachura J, Czarnywojtek A, et al. Management of the hormonal syndrome of neuroendocrine tumors. Arch Med Sci 2017; 13: 515-24.

2. Bast RC Jr, Hennessy B, Mills GB. The biology of ovarian cancer: new opportunities for translation. Nat Rev Cancer 2009; 9: 415-28.

3. Chambers AF, Groom AC, MacDonald IC. Dissemination and growth of cancer cells in metastatic sites. Nat Rev Cancer 2002; 2: 563-72.

4. Siegel R, Naishadham D, Jemal A. Cancer statistics, 2013. CA Cancer I Clin 2013; 63: 11-30.

5. Ancuceanu RV, Istudor V. Pharmacologically active natural compounds for lung cancer. Altern Med Rev 2004; 9: 402-19.

6. Feng Y, Wang N, Zhu M, Feng Y, Li H, Tsao S. Recent progress on anticancer candidates in patents of herbal medicinal products. Recent Pat Food Nutr Agric 2011; 3: 30-48.

7. Pu CY, Lan VM, Lan CF, Lang HC. The determinants of traditional Chinese medicine and acupuncture utilization for cancer patients with simultaneous conventional treatment. Eur J Cancer Care (Engl) 2008; 17: 340-9. 
8. Yen Y, So S, Rose M, et al. Phase I/II study of PHY906/ capecitabine in advanced hepatocellular carcinoma. Anticancer Res 2009; 29: 4083-92.

9. Jung $Y$, Jerng $U$, Lee $S$. A systematic review of anticancer effects of radix astragali. Chin J Integr Med 2016; 22: 225-36.

10. Xin T, Zhang F, Jiang Q, et al. The inhibitory effect of a polysaccharide from Codonopsis pilosula on tumor growth and metastasis in vitro. Int J Biol Macromol 2012; 51: 788-93.

11. Shi L, Zhang G, Zheng Z, Lu B, Ji L. Andrographolide reduced VEGFA expression in hepatoma cancer cells by inactivating HIF-1alpha: the involvement of JNK and MTA1/HDCA. Chem Biol Interact 2017; 273: 228-36.

12. Li CY, Wang EQ, Cheng Y, Bao JK. Oridonin: an active diterpenoid targeting cell cycle arrest, apoptotic and autophagic pathways for cancer therapeutics. Int J Biochem Cell Biol 2011; 43: 701-4.

13. Guo W, Zheng P, Zhang J, Ming L, Zhou C, Zhang S. Oridonin suppresses transplant rejection by depleting $T$ cells from the periphery. Int Immunopharmacol 2013; 17: 1148-54.

14. Bu HQ, Liu DL, Wei WT, et al. Oridonin induces apoptosis in SW1990 pancreatic cancer cells via p53- and caspase-dependent induction of p38 MAPK. Oncol Rep 2014; 31: 975-82.

15. Hsieh TC, Wijeratne EK, Liang JY, Gunatilaka AL, Wu JM Differential control of growth, cell cycle progression, and expression of NF-kappaB in human breast cancer cells MCF-7, MCF-10A, and MDA-MB-231 by ponicidin and oridonin, diterpenoids from the chinese herb Rabdosia rubescens. Biochem Biophys Res Commun 2005; 337: 224-31.

16. Zhou GB, Chen SJ, Wang ZY, Chen Z. Back to the future of oridonin: again, compound from medicinal herb shows potent antileukemia efficacies in vitro and in vivo. Cell Res 2007; 17: 274-6.

17. Huang J, Wu L, Tashiro S, Onodera S, Ikejima T. Reactive oxygen species mediate oridonin-induced HepG2 apoptosis through p53, MAPK, and mitochondrial signaling pathways. J Pharmacol Sci 2008; 107: 370-9.

18. Kang N, Zhang JH, Qiu F, Tashiro S, Onodera S, Ikejima T. Inhibition of EGFR signaling augments oridonin-induced apoptosis in human laryngeal cancer cells via enhancing oxidative stress coincident with activation of both the intrinsic and extrinsic apoptotic pathways. Cancer Lett 2010; 294: 147-58.

19. Chen S, Gao J, Halicka HD, Huang X, Traganos F, Darzynkiewicz Z. The cytostatic and cytotoxic effects of oridonin (Rubescenin), a diterpenoid from Rabdosia rubescens, on tumor cells of different lineage. Int J Oncol 2005; 26: 579-88.

20. Ma S, Tan W, Du B, et al. Oridonin effectively reverses cisplatin drug resistance in human ovarian cancer cells via induction of cell apoptosis and inhibition of matrix metalloproteinase expression. Mol Med Rep 2016; 13: 3342-8.

21. Wang S, Zhong Z, Wan J, et al. Oridonin induces apoptosis, inhibits migration and invasion on highly-metastatic human breast cancer cells. Am J Chin Med 2013 41: 177-96.

22. Dong Y, Zhang T, Li J, et al. Oridonin inhibits tumo growth and metastasis through anti-angiogenesis by blocking the Notch signaling. PLoS One 2014; 9: e113830.

23. Xia S, Zhang X, Li C, Guan H. Oridonin inhibits breast cancer growth and metastasis through blocking the Notch signaling. Saudi Pharm J 2017; 25: 638-643.
24. Liu OO, Chen K, Ye O, Jiang XH, Sun YW. Oridonin inhibits pancreatic cancer cell migration and epithelial-mesenchymal transition by suppressing Wnt/beta-catenin signaling pathway. Cancer Cell Int 2016; 16: 57.

25. Fontenot JD, Rudensky AY. A well adapted regulatory contrivance: regulatory $T$ cell development and the forkhead family transcription factor Foxp3. Nat Immunol 2005; 6: 331-7.

26. Hori S, Nomura T, Sakaguchi S. Control of regulatory $T$ cell development by the transcription factor Foxp3. Science 2003; 299: 1057-61.

27. Hori S, Sakaguchi S. Foxp3: a critical regulator of the development and function of regulatory $T$ cells. Microbes Infect 2004; 6: 745-51.

28. Fontenot JD, Rasmussen JP, Williams LM, Dooley J, Farr AG, Rudensky AY. Regulatory T cell lineage specification by the forkhead transcription factor foxp3. Immunity $2005 ; 22: 329-41$

29. Triulzi T, Tagliabue E, Balsari A, Casalini P. FOXP3 expression in tumor cells and implications for cancer progression. J Cell Physiol 2013; 228: 30-5.

30. Karanikas V, Speletas M, Zamanakou M, et al. Foxp3 expression in human cancer cells. J Transl Med 2008, 6: 19

31. Wang XR, Luo H, Li HL, et al. Overexpressed let-7a inhibits glioma cell malignancy by directly targeting K-ras, independently of PTEN. Neuro Oncol 2013; 15: 1491-501.

32. Chen GY, Chen C, Wang L, Chang X, Zheng P, Liu Y. Cutting edge: broad expression of the FoxP3 locus in epithelial cells: a caution against early interpretation of fatal inflammatory diseases following in vivo depletion of FoxP3-expressing cells. J Immunol 2008; 180: 5163-6.

33. Wang L, Liu R, Li W, et al. Somatic single hits inactivate the X-linked tumor suppressor FOXP3 in the prostate. Cancer Cell 2009; 16: 336-46.

34. Zuo T, Wang L, Morrison C, et al. FOXP3 is an X-linked breast cancer suppressor gene and an important repressor of the HER-2/ErbB2 oncogene. Cell 2007; 129: 1275-86.

35. Jiang G, Liu J, Ren B, et al. Anti-tumor and chemosensitization effects of Cryptotanshinone extracted from Salvia miltiorrhiza Bge. on ovarian cancer cells in vitro. J Ethnopharmacol 2017; 205: 33-40.

36. Zhang L, Ren B, Zhang J, et al. Anti-tumor effect of Scutellaria barbata D. Don extracts on ovarian cancer and its phytochemicals characterisation. J Ethnopharmacol 2017; 206: 184-92.

37. Zhang L, Zhang J, Qi B, et al. The anti-tumor effect and bioactive phytochemicals of Hedyotis diffusa willd on ovarian cancer cells. J Ethnopharmacol 2016; 192: 132-9.

38. Jiang G, Liu J, Ren B, et al. Anti-tumor effects of osthole on ovarian cancer cells in vitro. J Ethnopharmacol 2016; 193: 368-76.

39. Zhang L, Zhao Y. The regulation of Foxp3 expression in regulatory CD4(+)CD25(+)T cells: multiple pathways on the road. J Cell Physiol 2007; 211: 590-7.

40. Tan B, Anaka M, Deb S, et al. FOXP3 over-expression inhibits melanoma tumorigenesis via effects on proliferation and apoptosis. Oncotarget 2014; 5: 264-76.

41. Yang S, Liu Y, Li MY, et al. FOXP3 promotes tumor growth and metastasis by activating Wnt/beta-catenin signaling pathway and EMT in non-small cell lung cancer. Mol Cancer 2017; 16: 124.

42. Zhang $\mathrm{C}, \mathrm{Xu} \mathrm{Y}, \mathrm{Hao} \mathrm{Q}$, et al. FOXP3 suppresses breast cancer metastasis through downregulation of CD44. Int J Cancer 2015; 137: 1279-90. 
43. Sauer S, Bruno L, Hertweck A, et al. T cell receptor signaling controls Foxp3 expression via PI3K, Akt, and mTOR. Proc Natl Acad Sci USA 2008; 105: 7797-802.

44. Delgoffe GM, Kole TP, Zheng Y, et al. The mTOR kinase differentially regulates effector and regulatory $T$ cell lineage commitment. Immunity 2009; 30: 832-44.

45. Ouyang W, Beckett O, Ma Q, Paik JH, DePinho RA, Li MO. Foxo proteins cooperatively control the differentiation of Foxp3+ regulatory T cells. Nat Immunol 2010; 11: 618-27.

46. Merkenschlager M, von Boehmer H. PI3 kinase signalling blocks Foxp 3 expression by sequestering Foxo factors. J Exp Med 2010; 207: 1347-50. 